\title{
Fundamental Study toward Improving the Performance of a High-Moisture Biomass-Fueled Redox Flow Fuel Cell
}

\author{
Wei Liu, ${ }^{\dagger}$ Yutao Gong, ${ }^{\dagger}$ Andrew Tricker, ${ }^{\dagger}$ Gaoxiang Wu, ${ }^{\dagger}$ Congmin Liu, $"$ Zi-sheng Chao, ${ }^{t} s$ \\ and Yulin Deng, *广
}

$\nmid$ School of Chemical \& Biomolecular Engineering and RBI, Georgia Institute of Technology 500 10th Street N.W., Atlanta, GA 30332, USA

* State Key Laboratory of Chemo/Biosensing and Chemometrics, College of Chemistry and Chemical Engineering, Hunan University, Changsha 410082, China

${ }^{\S}$ College of Materials Science and Engineering, Changsha University of Science and Technology, Changsha, Hunan 410114, China

" National Institute of Clean-and-Low-Carbon Energy, Beijing 102211, China 


\section{Supporting information}

\section{Supporting Figures}

\section{A}
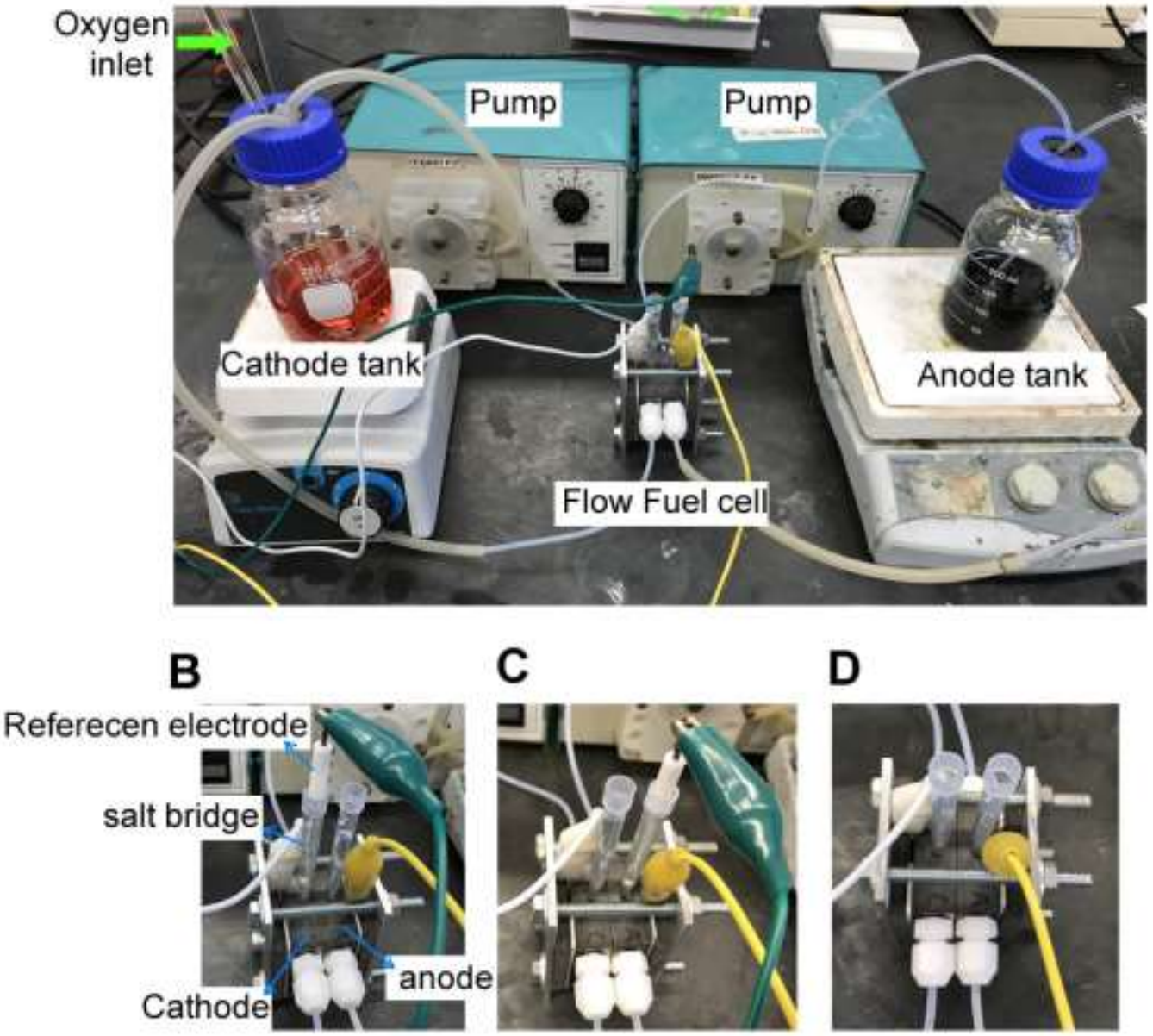

C

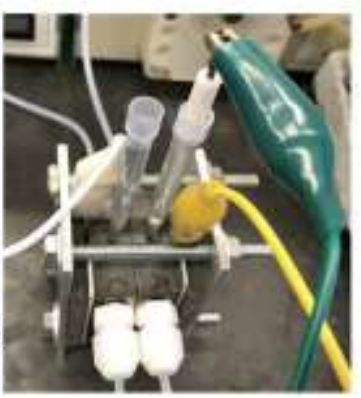

D

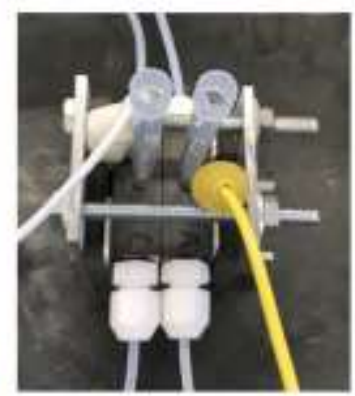

Fig. S1 (A) The photograph of the flow fuel cell experimental setup before running; (B)

Three-electrode configuration for cathodic in-situ electrochemical measurement; (C)

Three-electrode configuration for anodic in-situ electrochemical measurement; (D)

Two-electrode configuration for the flow fuel cell performance testing. 

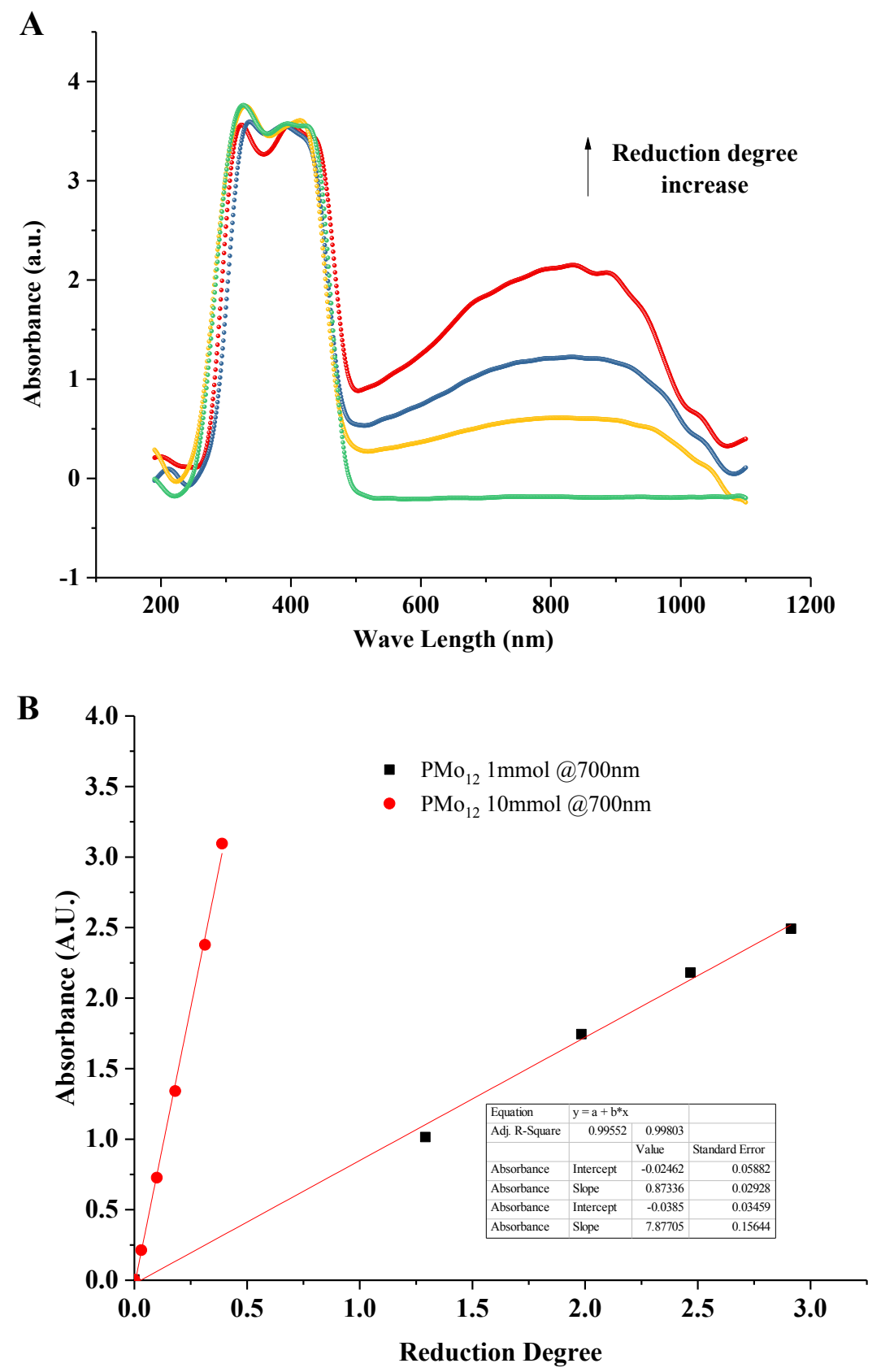

Fig. S2 (A) UV-VIs spectrum of PMo 12 solutions with different reduction degrees (measured in concentration $1 \mathrm{mmol} \mathrm{l}^{-1}$ ); (B) Linear relationship of absorbance of UV-Vis spectrum and reduction degree of $\mathrm{PMo}_{12}$. 

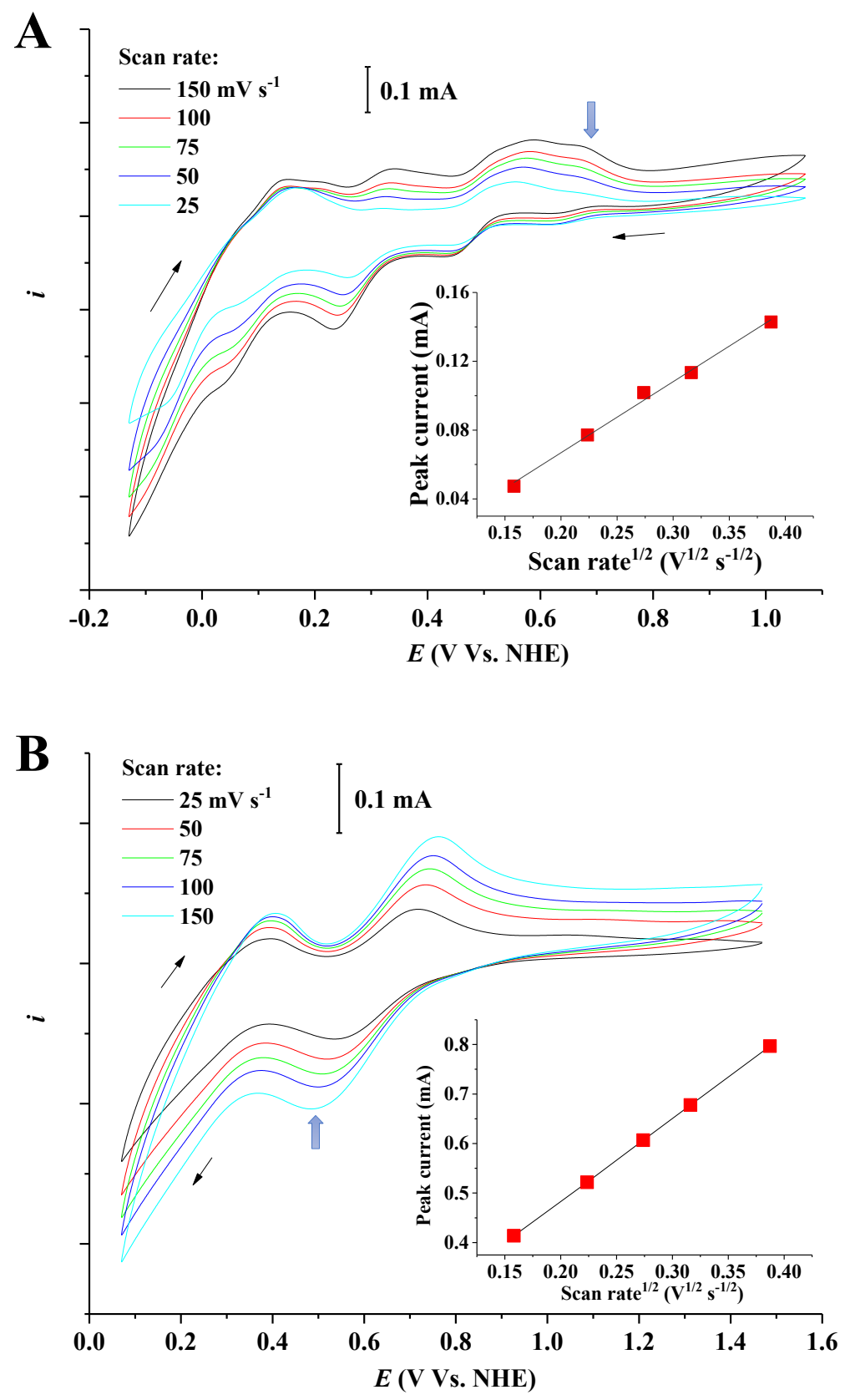

Fig. S3 CV curves of $\mathrm{PMo}_{12}$ (A) and $\mathrm{PMoV}$ (B) solution in different scan rates. The concentration of POM solutionis $0.01 \mathrm{~mol} \mathrm{l}^{-1}$. The inset figures are plots of the peak current (noted with arrow) vs. Scan rate ${ }^{1 / 2}$. 

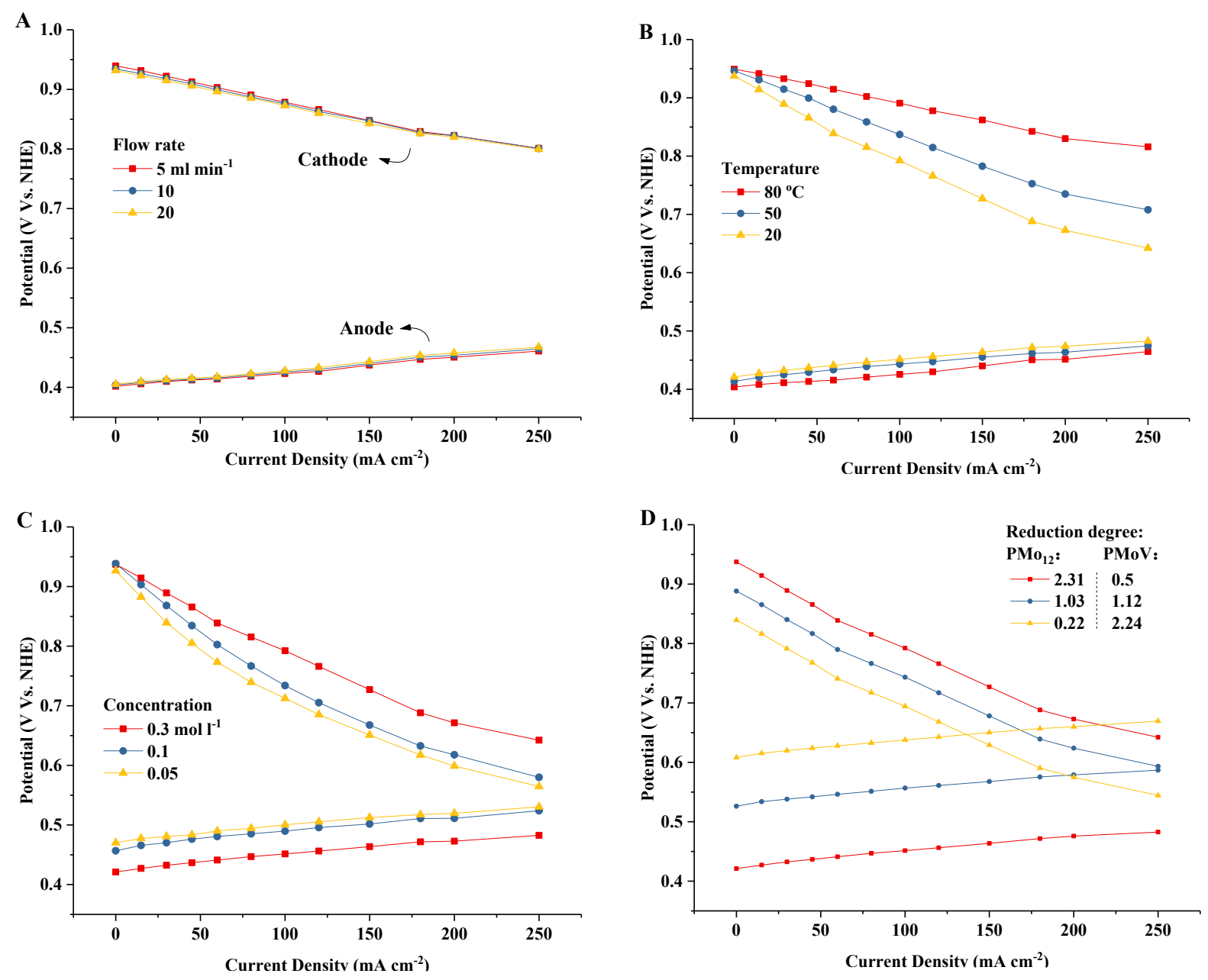

Fig. S4 Working potentials of anode and cathode in constant current discharging under the conditions of different flow rates, fixed at temperature $80{ }^{\circ} \mathrm{C}$, concentration $0.3 \mathrm{~mol} \mathrm{l}^{-1}$ and reduction degree of $\mathrm{PMo}_{12}$ at 2.2, $\mathrm{PMoV}$ at 0.5 (A); different temperatures, fixed at flow rate 20 $\mathrm{ml} \mathrm{min}{ }^{-1}$, concentration $0.3 \mathrm{~mol} \mathrm{l}^{-1}$ and reduction degree of $\mathrm{PMo}_{12}$ at $2.2, \mathrm{PMoV}$ at 0.5 (B); different concentration of POM solutions, fixed at temperature $20{ }^{\circ} \mathrm{C}$, flow rate $20 \mathrm{ml} \mathrm{min}^{-1}$ and reduction degree of $\mathrm{PMo}_{12}$ at 2.2, $\mathrm{PMoV}$ at $0.5(\mathrm{C})$; and different reduction degrees of $\mathrm{PMo}_{12}$ and PMoV, fixed at temperature $20{ }^{\circ} \mathrm{C}$, concentration $0.3 \mathrm{~mol} \mathrm{l}^{-1}$ and flow rate $20 \mathrm{ml} \mathrm{min}^{-1}$ (D). 

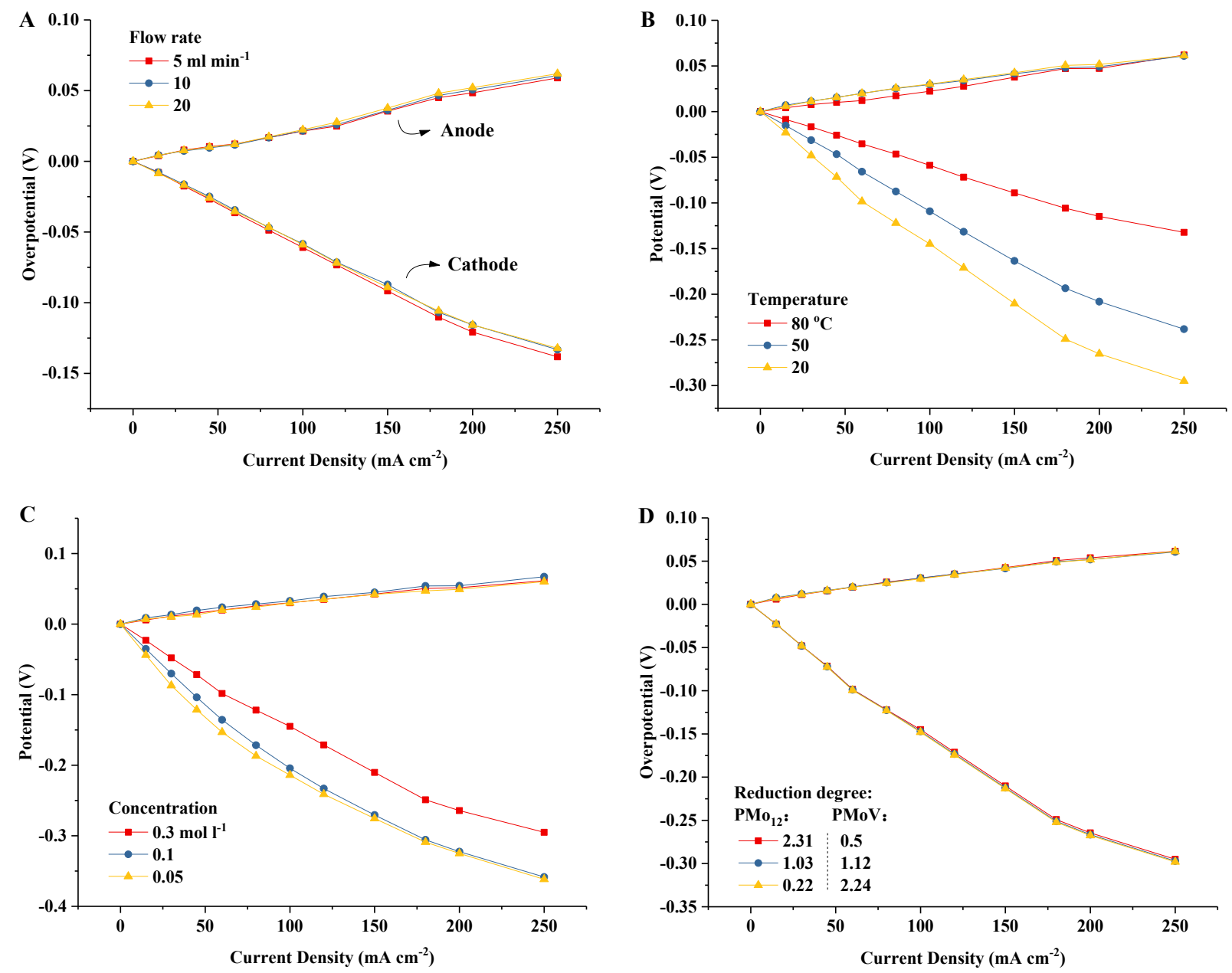

Fig. S5 Overpotentials of anode and cathode in constant current discharging under the conditions of different flow rates, fixed at temperature $80{ }^{\circ} \mathrm{C}$, concentration $0.3 \mathrm{~mol} \mathrm{l}^{-1}$ and reduction degree of $\mathrm{PMo}_{12}$ at 2.2, $\mathrm{PMoV}$ at 0.5 (A); different temperatures, fixed at flow rate $20 \mathrm{ml} \mathrm{min}{ }^{-1}$, concentration $0.3 \mathrm{~mol} \mathrm{l}^{-1}$ and reduction degree of $\mathrm{PMo}_{12}$ at 2.2 , $\mathrm{PMoV}$ at 0.5 (B); different concentration of POM solutions, fixed at temperature $20{ }^{\circ} \mathrm{C}$, flow rate $20 \mathrm{ml} \mathrm{min}^{-1}$ and reduction degree of $\mathrm{PMo}_{12}$ at 2.2, $\mathrm{PMoV}$ at $0.5(\mathrm{C})$; and different reduction degrees of $\mathrm{PMo}_{12}$ and $\mathrm{PMoV}$, fixed at temperature $20{ }^{\circ} \mathrm{C}$, concentration $0.3 \mathrm{~mol} \mathrm{l}^{-1}$ and flow rate $20 \mathrm{ml} \mathrm{min}^{-1}$ (D). 

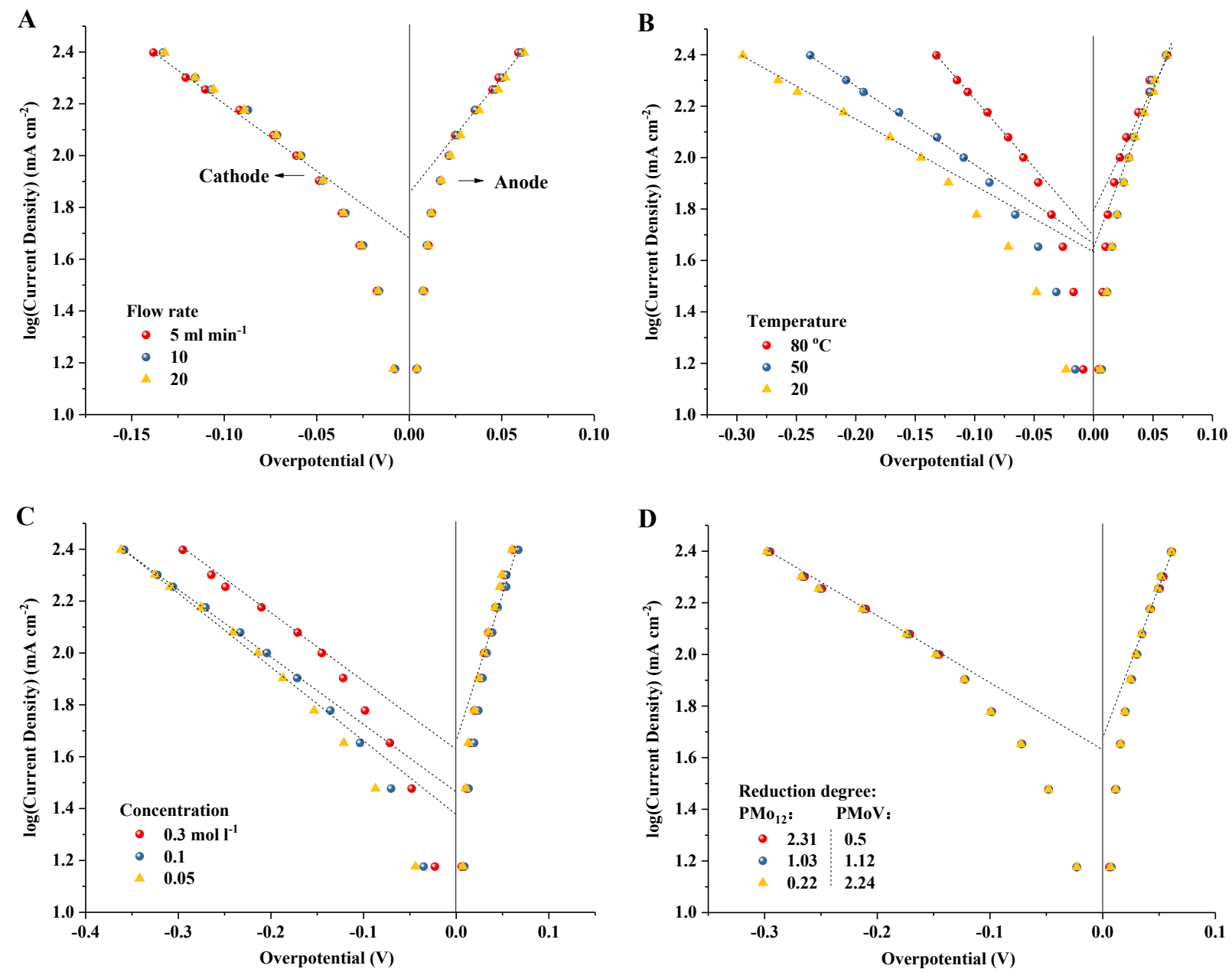

Fig. S6 Tafel plots at anode and cathode in constant current discharging under the conditions of different flow rates, fixed at temperature $80^{\circ} \mathrm{C}$, concentration $0.3 \mathrm{~mol} \mathrm{l}^{-1}$ and reduction degree of $\mathrm{PMo}_{12}$ at $2.2, \mathrm{PMoV}$ at 0.5 (A); different temperatures, fixed at flow rate $20 \mathrm{ml} \mathrm{min}^{-1}$, concentration $0.3 \mathrm{~mol} \mathrm{l}^{-1}$ and reduction degree of $\mathrm{PMo}_{12}$ at 2.2, $\mathrm{PMoV}$ at 0.5 (B); different concentration of POM solutions, fixed at temperature $20{ }^{\circ} \mathrm{C}$, flow rate $20 \mathrm{ml} \mathrm{min}^{-1}$ and reduction degree of $\mathrm{PMo}_{12}$ at 2.2, PMoV at $0.5(\mathrm{C})$; and different reduction degrees of $\mathrm{PMo}_{12}$ and $\mathrm{PMoV}$, fixed at temperature $20{ }^{\circ} \mathrm{C}$, concentration $0.3 \mathrm{~mol} \mathrm{l}^{-1}$ and flow rate $20 \mathrm{ml} \mathrm{min}^{-1}$ (D) 

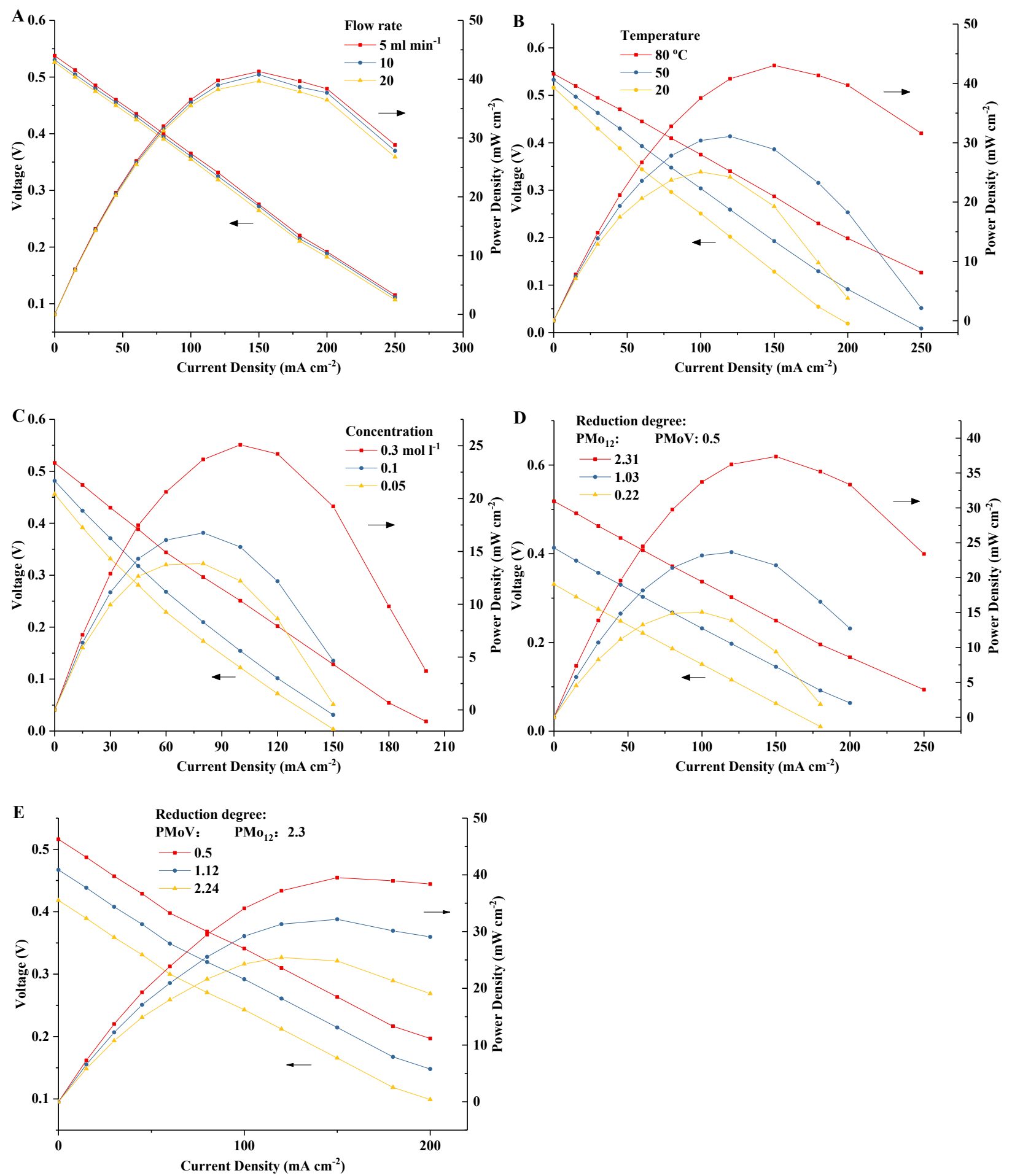

Fig. S7 Voltage-current density and power density-current density curves of flow fuel cell in constant current discharging under the conditions of different flow rates, fixed at temperature 80 
${ }^{\circ} \mathrm{C}$, concentration $0.3 \mathrm{~mol} \mathrm{l}^{-1}$ and reduction degree of $\mathrm{PMo}_{12}$ at 2.2 , PMoV at 0.5 (A); different temperatures, fixed at flow rate $20 \mathrm{ml} \mathrm{min}{ }^{-1}$, concentration $0.3 \mathrm{~mol} \mathrm{l}^{-1}$ and reduction degree of $\mathrm{PMo}_{12}$ at 2.2, PMoV at $0.5(\mathrm{~B})$; different concentration of POM solutions, fixed at temperature $20{ }^{\circ} \mathrm{C}$, flow rate $20 \mathrm{ml} \mathrm{min}^{-1}$ and reduction degree of $\mathrm{PMo}_{12}$ at 2.2, $\mathrm{PMoV}$ at $0.5(\mathrm{C})$; different reduction degrees of $\mathrm{PMo}_{12}$, fixed at $\mathrm{PMoV}$ reduction of 0.5 , temperature $20{ }^{\circ} \mathrm{C}$, concentration

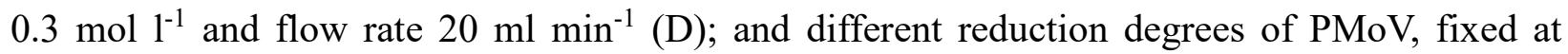
$\mathrm{PMo}_{12}$ reduction of 2.3 , temperature $20{ }^{\circ} \mathrm{C}$, concentration $0.3 \mathrm{~mol} \mathrm{l}^{-1}$ and flow rate $20 \mathrm{ml} \mathrm{min}^{-1}$ (E). 

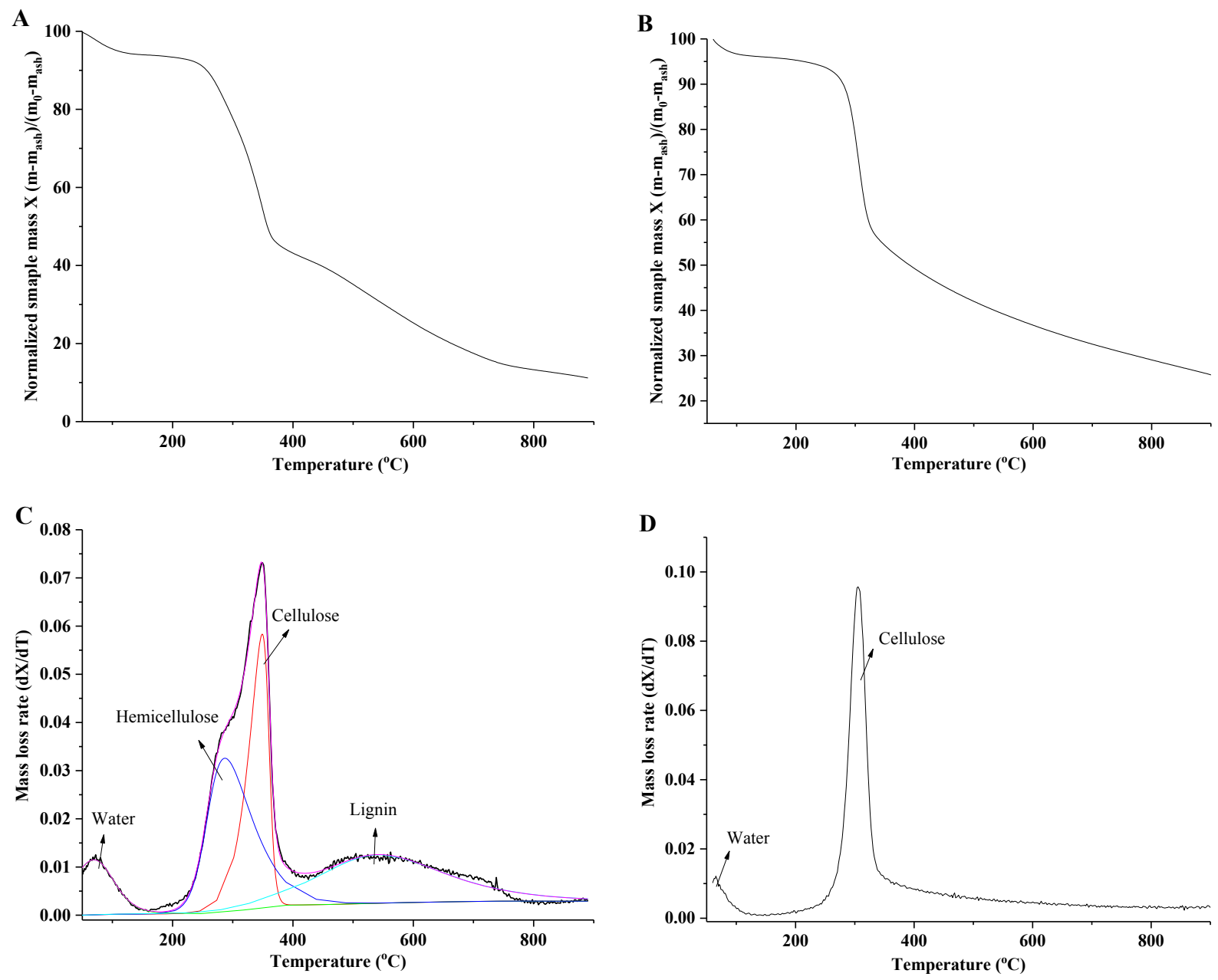

Fig. S8 (A) TGA of initial wheat straw; (B) TGA of wheat straw residue after reacted with PMo12 for 4 hours; (C) theoretical one-step devolatilization model analysis of fig (A); (D) differential analysis of fig (B). 


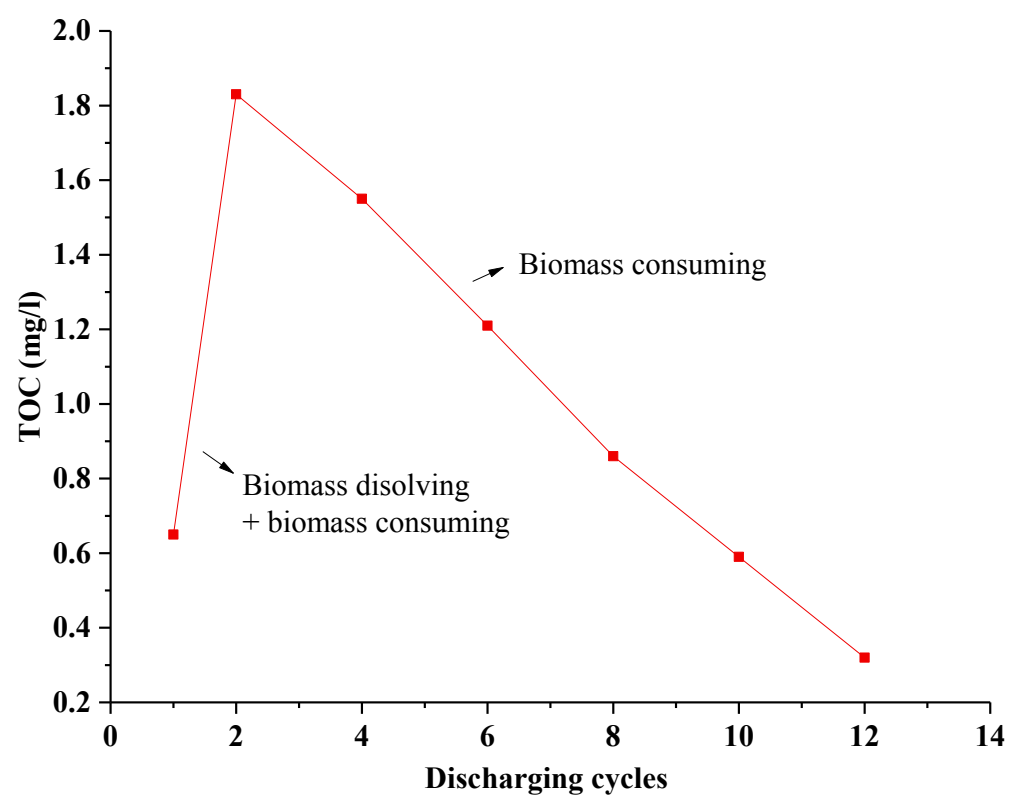

Fig. S9 Total organic carbon (TOC) analysis of carbon content in the anolyte (wheat straw $+\mathrm{PMo}_{12}$ ) solution with different repeated discharging cycles. For each cycle, firstly wheat straw and $\mathrm{PMo}_{12}$ solution was heated and refluxed for 4 hours (condition: initial wheat straw adding at $1^{\text {st }}$ time: $0.5 \mathrm{~g}$; PMo12: $0.3 \mathrm{~mol} \mathrm{l}^{-1}$; total volume of anolyte solution: $50 \mathrm{ml}$ ), then was discharged in redox fuel cell until the current density was lower than $10^{-3} \mathrm{~A} \mathrm{~cm}^{-2}$. 


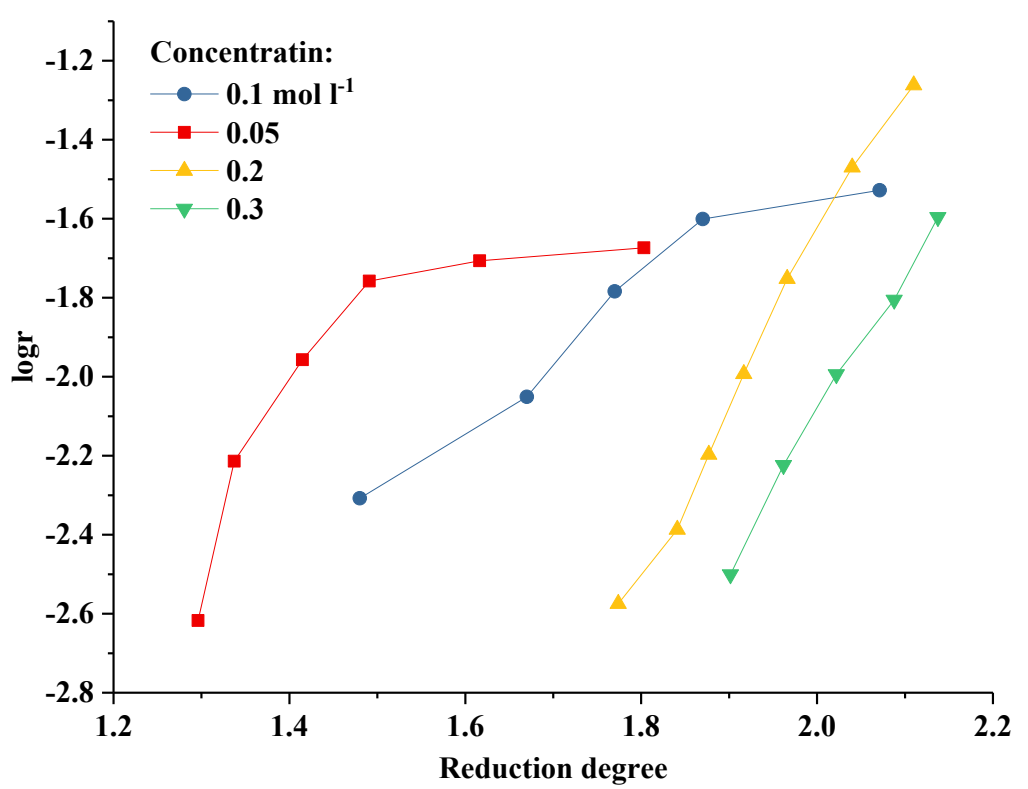

Fig. S10 Relationship of electron transfer rate from acid form of PMoV to oxygen vs. Reduction degree. 


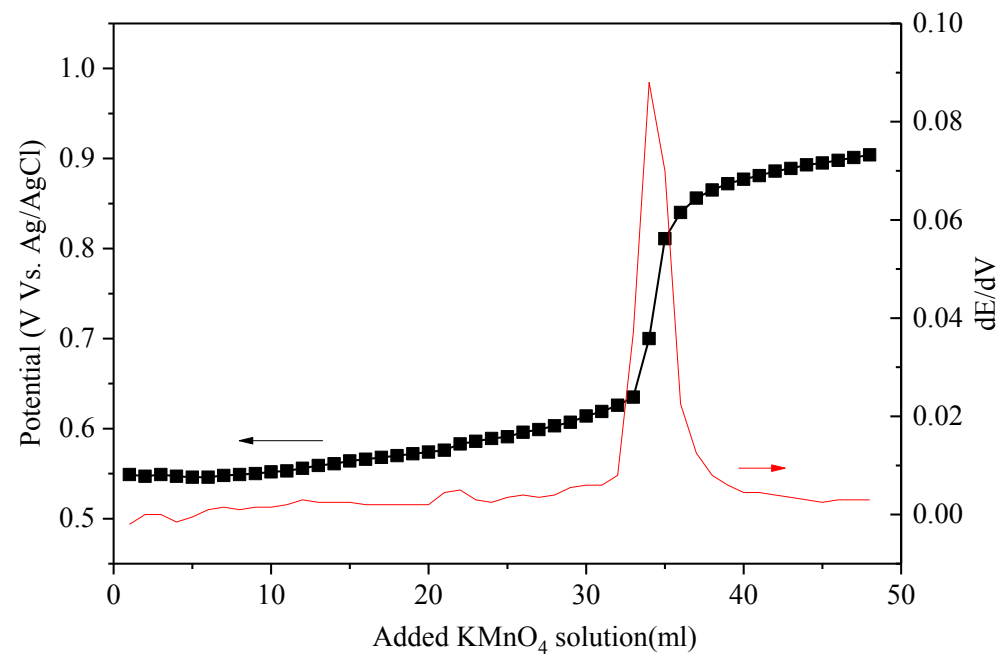

Fig. S11 A typic potentiometric titration curve (black dot line) and the corresponding differential curve (red line) for determining the reduction degree of $\mathrm{PMoV}$ by $\mathrm{KMnO}_{4}$ solution. The titration method was described in method section 2.5 in manuscript. 

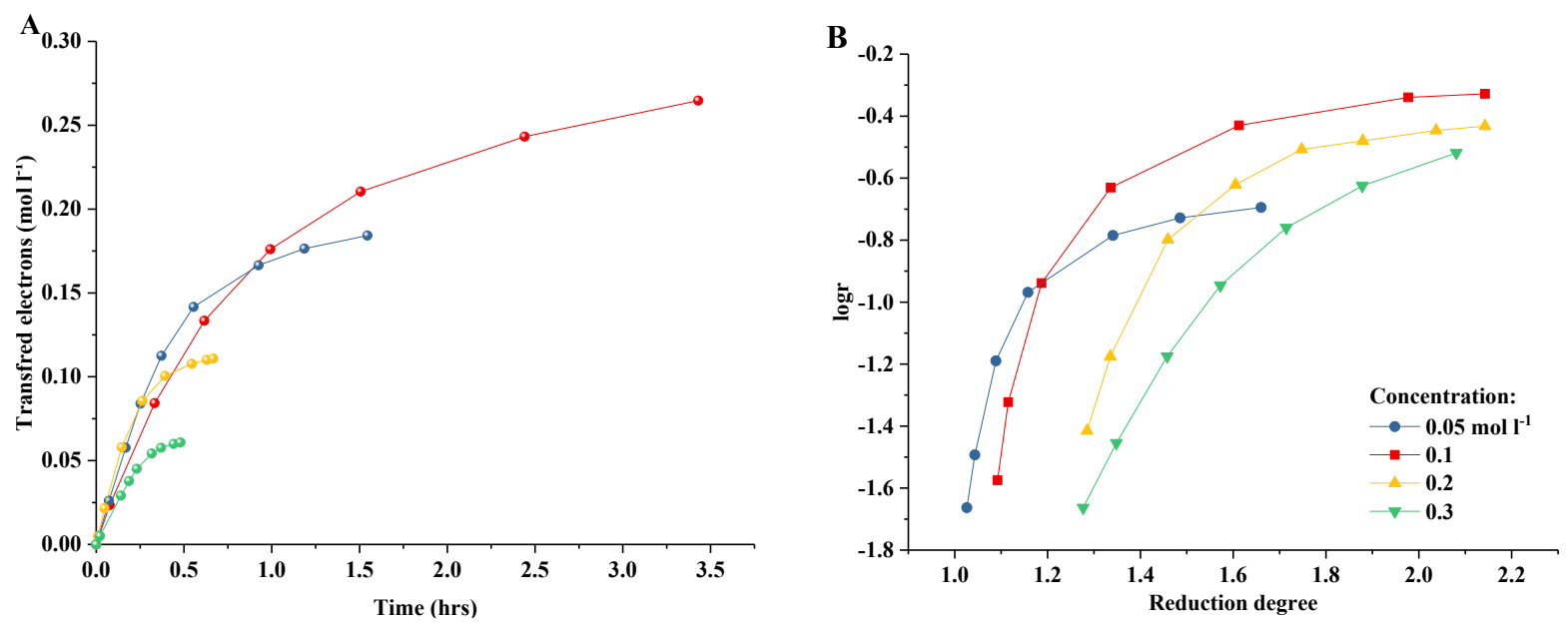

Fig. S12 (A) Electrons transferred from $\mathrm{Na}_{2}-\mathrm{PMoV}$ to oxygen over different reaction time; (B)

Relationship of electron transfer rate from $\mathrm{Na}_{2}-\mathrm{PMoV}$ to oxygen vs. Reduction degree 


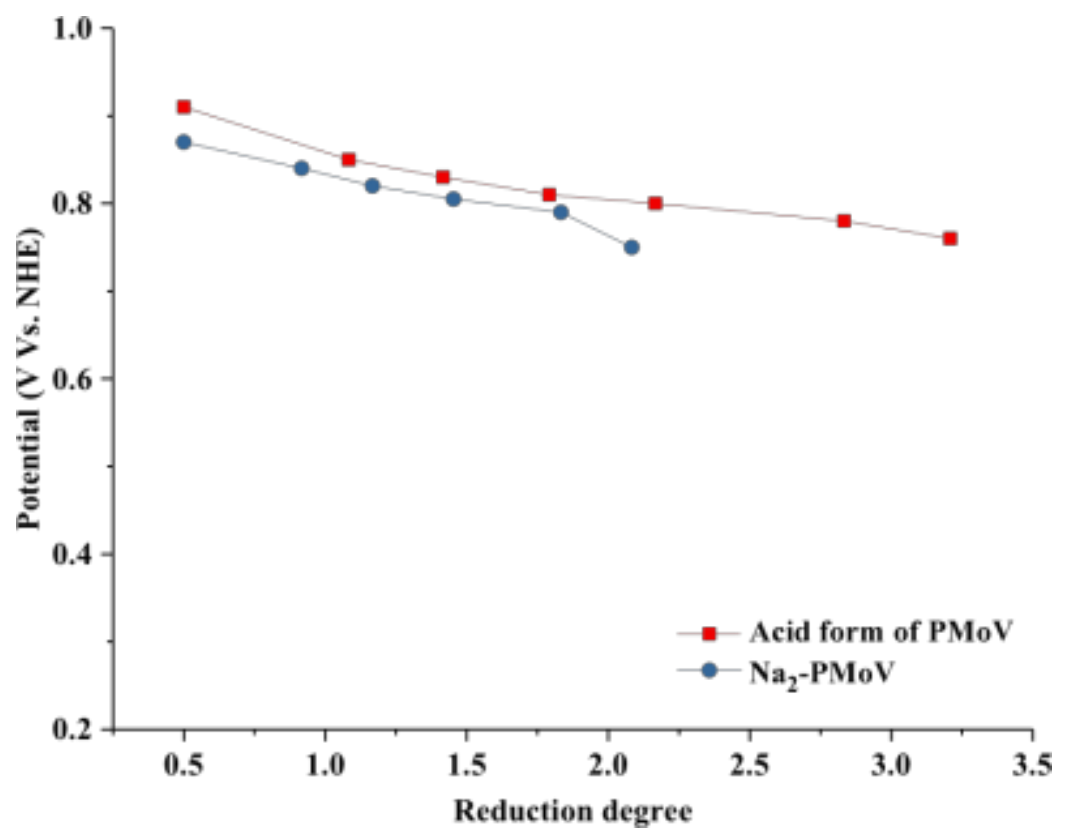

Fig. S13 Electrode potentials of acid form of PMoV and $\mathrm{Na}_{2}-\mathrm{PMoV}$. 


\section{Supporting Tables}

Table S1 Kinetic parameters in Tafel plots of $\mathrm{PMo}_{12}$ and PMoV under different conditions

\begin{tabular}{|c|c|c|c|c|}
\hline \multirow[b]{2}{*}{ Conditions } & \multicolumn{2}{|c|}{ Anode-POM-1 } & \multicolumn{2}{|c|}{ Cathode-POM-2 } \\
\hline & Tafel slop (b) & 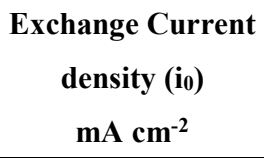 & Tafel slop (b) & $\begin{array}{c}\text { Exchange Current } \\
\text { density (io) } \\
\text { mA cm }\end{array}$ \\
\hline Flow rate $5 \mathrm{ml} \mathrm{min}^{-1}$ & 0.113 & 71.6 & -0.195 & 47.5 \\
\hline Flow rate $10 \mathrm{ml} \mathrm{min}^{-1}$ & 0.113 & 71.6 & -0.195 & 47.5 \\
\hline Flow rate $20 \mathrm{ml} \mathrm{min}^{-1}$ & 0.113 & 71.6 & -0.195 & 47.5 \\
\hline Temperature $20^{\circ} \mathrm{C}$ & 0.085 & 45.1 & -0.381 & 43.3 \\
\hline Temperature $50^{\circ} \mathrm{C}$ & 0.085 & 45.1 & -0.327 & 46.4 \\
\hline Temperature $80^{\circ} \mathrm{C}$ & 0.103 & 62.4 & -0.189 & 49.4 \\
\hline Concentration $0.05 \mathrm{~mol} \mathrm{l}^{-1}$ & 0.089 & 46.6 & -0.353 & 23.6 \\
\hline Concentration $0.05 \mathrm{~mol} \mathrm{l}^{-1}$ & 0.089 & 46.6 & -0.387 & 29.1 \\
\hline Concentration $0.05 \mathrm{~mol} \mathrm{l}^{-1}$ & 0.089 & 46.6 & -0.381 & 42.5 \\
\hline Reduction degree $\mathrm{PMo}_{12} 2.31$ & 0.085 & 46.9 & & \\
\hline Reduction degree $\mathrm{PMo}_{12} 1.03$ & 0.085 & 46.9 & & \\
\hline Reduction degree $\mathrm{PMo}_{12} 0.22$ & 0.085 & 46.9 & & \\
\hline Reduction degree PMoV 0.5 & & & -0.381 & 42.5 \\
\hline Reduction degree PMoV 1.12 & & & -0.381 & 42.5 \\
\hline Reduction degree PMoV 2.24 & & & -0.381 & 42.5 \\
\hline
\end{tabular}


Table S2 Fitted values of elements in equivalent circuits

\begin{tabular}{|c|c|c|c|c|c|c|c|c|c|}
\hline & \multicolumn{3}{|c|}{ PMoV in cathode side } & & \multicolumn{4}{c|}{ PMo12 in anode side } \\
\hline & $\begin{array}{c}80^{\circ} \mathrm{C}-0.3 \\
\mathrm{M}\end{array}$ & $\begin{array}{c}25^{\circ} \mathrm{C}-0.3 \\
\mathrm{M}\end{array}$ & $\begin{array}{c}25^{\circ} \mathrm{C}-0.1 \\
\mathrm{M}\end{array}$ & $\begin{array}{c}25^{\circ} \mathrm{C}-0.05 \\
\mathrm{M}\end{array}$ & & $80^{\circ} \mathrm{C}-0.3$ \\
$\mathrm{M}$ & $\begin{array}{c}25^{\circ} \mathrm{C}-0.3 \\
\mathrm{M}\end{array}$ & $\begin{array}{c}25^{\circ} \mathrm{C}-0.1 \\
\mathrm{M}\end{array}$ & $\begin{array}{c}25^{\circ} \mathrm{C}-0.05 \\
\mathrm{M}\end{array}$ \\
\hline $\mathrm{Rs}$ & 0.0796 & 0.1081 & 0.0860 & 0.084 & $\mathrm{Rs}$ & 0.0458 & 0.0329 & 0.0335 & 0.0332 \\
\hline $\mathrm{R} 1$ & 0.0193 & 0.2611 & 0.8428 & 1.24 & $\mathrm{R} 1$ & 0.1774 & 0.2163 & 0.2062 & 0.1952 \\
\hline $\mathrm{C} 1$ & 0.1306 & 0.1189 & 0.0365 & 0.041 & $\mathrm{C} 2$ & 0.0007 & 0.0006 & 0.0006 & 0.0006 \\
\hline $\mathrm{R} 2$ & 0.426 & 0.735 & 0.836 & 0.838 & $\mathrm{~W} 1-$ & 0.0184 & 0.0196 & 0.102 & 0.7196 \\
\hline $\mathrm{C} 2$ & 0.00032 & 0.00021 & 0.00019 & 0.0002 & $\mathrm{~W} 1-\mathrm{T}$ & 0.871 & 1.702 & 6.409 & 17.76 \\
\hline W1- & 0.128 & 0.828 & 2.229 & 3.534 & $\mathrm{~W} 1-\mathrm{P}$ & 0.3791 & 0.3583 & 0.3629 & 0.3872 \\
\hline $\mathrm{R}$ & 3.818 & 19.110 & 29.121 & 21.97 & & & & & \\
\hline W1-T & 0.329 & 0.377 & 0.332 & 0.373 & & & & \\
\hline W1-P & & & & & & & \\
\hline
\end{tabular}


Table S3 GC-MS result of reaction solution of $\mathrm{PMo}_{12}$ with wheat straw

\begin{tabular}{|l|l|l|l|}
\hline Detected structures & Normalized & Detected structures & Normalized \\
\hline & percentage & & percentage \\
\hline & $11.84 \%$ & & $9.87 \%$ \\
\hline & & & \\
\hline & & & \\
\hline
\end{tabular}


Table S4 GC analysis in the emission gas of wheat straw fuel cell

\begin{tabular}{|c|c|}
\hline Measured gas & Percentage mol\% \\
\hline Hydrogen & 0 \\
\hline Nitrogen & 91.0 \\
\hline Oxygen & 5.2 \\
\hline Methane & 0 \\
\hline Mono carbon oxide & 0.01 \\
\hline Carbon dioxide & 3.8 \\
\hline
\end{tabular}

